\title{
Opinião de auxiliares e técnicos de enfermagem sobre a sistematização da assistência de enfermagem ${ }^{1}$
}

\section{Opinion of nursing aids and nursing technicians about the nursing process}

\section{Opinión de los auxiliares y técnicos de enfermería sobre el proceso de atención de enfermería}

Luciana Aparecida Ribeiro Ramos', Emília Campos de Carvalho", Silvia Rita Marin da Silva Canini ${ }^{\text {II }}$

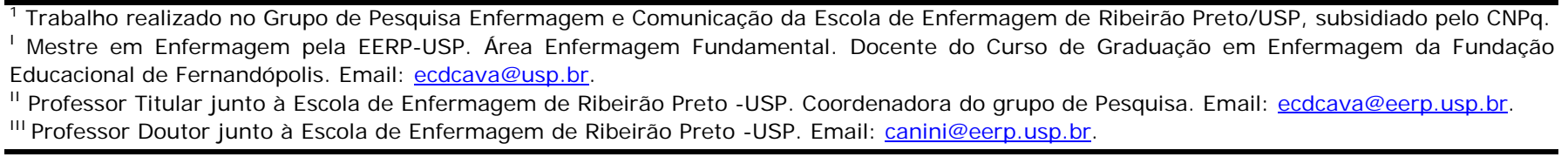

\section{RESUMO}

Este estudo teve como objetivo identificar a percepção de auxiliares e técnicos de enfermagem sobre sistematização da assistência de enfermagem e os aspetos legais de sua participação nesse processo, sobre a contribuição dessa metodologia na organização do serviço e atividades que realizam. Estudo descritivo, com 77 profissionais de um hospital escola do interior do Estado de São Paulo, em 2006. Os dados, coletados por meio de questionário e escala tipo Likert, revelaram que os profissionais reconhecem a utilização da SAE em seu setor de trabalho, mas não relacionam suas atividades ao método. A maioria referiu que contribui recepcionando o paciente na unidade, coletando dados como freqüência cardíaca, pressão arterial, temperatura, realizando as prescrições de enfermagem. Ainda, referem participar da avaliação comunicando as alterações observadas. Conclui-se que a participação dos auxiliares e técnicos de enfermagem nesta metodologia ainda é obscura, os mesmos não percebem suas atribuições, fato reforçado pela compreensão dúbia da legislação, evidenciando necessidade premente da inclusão deste tema na grade curricular dos cursos de auxiliares e técnicos de enfermagem, esclarecendo as atribuições e responsabilidades de cada membro da equipe de enfermagem.

Descritores: Processos de enfermagem; Cuidados de enfermagem; Planejamento da assistência ao paciente; Equipe de enfermagem.

\section{ABSTRACT}

This study aimed to identify the perception of nursing auxiliaries and technicians about nursing care systemization (NCS) and the legal aspects of their participation in this process, about the contribution of this method in the organization of care services and the activities they perform. This descriptive study involved 77 professionals at a teaching hospital in the countryside of São Paulo's state/Brazil, in 2006. The data, collected through a questionnaire and Likert-type scale, revealed that the professionals recognize the use of NCS in their work sector, but do not relate their activities to the method. Most professionals indicated that they contribute by receiving the patient at the unit, collecting data like heart frequency, blood pressure and temperature, and by elaborating nursing prescriptions. They also mention participating in assessment, communicating the changes observed. It is concluded that nursing auxiliaries' and technicians' participation in this method remains obscure, that they do not perceive their attributions. This fact is strengthening by the biased understanding of legislation, evidencing the urgent need to include this theme in the curriculum of nursing auxiliary and technician courses, clarifying the attributions and responsibilities of each nursing team member.

Descriptors: Nursing process; Nursing care; Patient care planning; Nursing team.

\section{RESUMEN}

La finalidad de este estudio fue identificar la percepción de auxiliares y técnicos de enfermería sobre la sistematización de la atención de enfermería y los aspectos legales de su participación en este proceso, sobre la contribución de esa metodología en la organización del servicio y las actividades realizadas por estos profesionales. Se trata de un estudio descriptivo, con 77 profesionales de un hospital-escuela del interior del Estado de São Paulo, Brasil, en 2006. Los datos, recolectados mediante cuestionario y escala del tipo Likert, revelaron que los profesionales reconocen la utilización de SAE en su sector de trabajo, pero no relacionan sus actividades con el método. La mayoría indica que contribuye recibiendo el paciente en la unidad, recolectando datos como frecuencia cardíaca, presión arterial, temperatura, realizando las prescripciones de enfermería. Además, refieren participar de la evaluación, comunicando las alteraciones observadas. Se concluye que la participación de los auxiliares y técnicos de enfermería en esta metodología todavía es obscura, no perciben sus atribuciones. Este hecho es reforzado por la comprensión dudosa de la legislación, evidenciando necesidad urgente de incluir este tema en el currículo de los cursos de auxiliares y técnicos de enfermería, aclarando las atribuciones y responsabilidades de cada miembro del equipo de enfermería.

Descriptores: Procesos de enfermería; Atención de enfermería; Planificación de atención al paciente; Grupo de enfermería. 


\section{NTRODUÇÃO}

O surgimento do cuidado de enfermagem sistematizado deu-se em função da necessidade da profissão definir seu corpo de conhecimento próprio e fundamentar sua ação com base em conceitos e teorias que permitiriam a tomada de decisão relacionada aos aspectos da assistência de enfermagem $^{(1)}$; foi ainda influenciado pela ênfase dada ao método de solução de problemas, cujas raízes se alicerçam no método científico ${ }^{(2)}$.

$\mathrm{Na}$ literatura internacional, Processo de Enfermagem (PE) é considerado como a forma mais usual de organizar a assistência de Enfermagem; no Brasil, recebe diferentes denominações: Sistematização da Assistência, Metodologia da Assistência, Planejamento da Assistência, Processo do Cuidado, Metodologia do Cuidado, Processo de Assistência, Consulta de Enfermagem, Processo de Atenção em Enfermagem e Processo de Enfermagem ${ }^{(3-4)}$.

A denominação Sistematização da Assistência de Enfermagem (SAE) ficou conhecida como sinônimo de $P E$, uma vez que o COFEN ${ }^{(5)}$ sugeriu as fases deste último como obrigatórias para Sistematização da Assistência; dada a divulgação desta nomeação entre os membros da equipe de enfermagem, no Brasil, esse termo foi acolhido no presente estudo.

A incorporação da SAE no cenário da prática da enfermagem brasileira teve início em 1999 com a decisão do Conselho Regional de Enfermagem COREN-SP/DIR/ 008/99(6), tornando obrigatória a sua implantação em todas as instituições públicas e privadas que oferecem serviço de enfermagem no âmbito do estado de São Paulo; posteriormente, em 2002, o Conselho Federal de Enfermagem, por meio da resolução do COFEN 272/2002 ${ }^{(5)}$, ampliou a exigência para todo território brasileiro.

Apesar de haver diversidade de funções previstas em $\operatorname{Lei}^{(7)}$, no que se refere à complexidade da assistência a ser prestada, na prática, constata-se que apenas o enfermeiro possui atribuições diferenciadas. De maneira geral, as atribuições específicas do enfermeiro, e que o diferenciam, são funções administrativas e de supervisão da assistência. Mesmo os cuidados mais complexos, prescritos por lei, geralmente não são prestados por ele.

O ensino da SAE é proporcionado aos graduandos de enfermagem; entretanto, pouca ou nenhuma informação é dada durante a formação dos profissionais de nível médio em função da dificuldade em delimitar as competências de cada membro da equipe de enfermagem no contexto da operacionalização dessa metodologia ${ }^{(3)}$.

Contudo, a literatura aponta dificuldades para a implantação desse método na enfermagem brasileira $^{(4)}$. Um desses entraves diz respeito à constituição da equipe de enfermagem, com profissionais de diferentes níveis educacionais e competências legais, mas, nem sempre ficam evidentes as atividades específicas de cada um deles. Em muitas situações o enfermeiro assume majoritariamente atividades burocrático-gerenciais enquanto os auxiliares e técnicos de enfermagem prestam a assistência ao paciente ${ }^{(8-9)}$. Outro aspecto é o desconhecimento, por parte dos auxiliares e técnicos de enfermagem, sobre seu papel e contribuição nesse processo de trabalho. Soma-se a este aspecto a perda de especificidade do próprio trabalho da equipe de enfermagem, uma vez que o cuidado ao paciente é prerrogativa de toda equipe de saúde ${ }^{(9)}$.

Desta forma, visando a contribuir na reflexão sobre a inserção de auxiliares e técnicos de enfermagem nessa metodologia, a partir da percepção desses profissionais, desenvolveu-se este estudo com o objetivo de caracterizar o processo de trabalho dos auxiliares e técnicos de enfermagem quanto aos seguintes aspectos da SAE: informação sobre esta metodologia durante sua formação profissional; informação sobre este método de trabalho no setor onde trabalha; informação quanto à aprovação legal para participarem da mesma; percepção de sua participação nas fases dessa sistematização da assistência; auxílio na organização do seu serviço assistencial; atividades realizadas por estes profissionais.

\section{PROCEDI MENTOS METODOLÓGI COS}

Este estudo de natureza descritiva foi realizado em um hospital escola do interior paulista, nos três setores em que a Sistematização da Assistência de Enfermagem está implantada.

Foram considerados como critérios de inclusão: estar trabalhando nos dias da coleta de dados, pertencer às referidas unidades e concordar em participar da investigação. Dos 120 sujeitos elegíveis, 77 responderam os instrumentos de coleta. O recrutamento dos sujeitos ocorreu com base na escala de folga mensal de enfermagem do mês de outubro de 2006, a qual foi fornecida pela diretoria de enfermagem do referido hospital.

Para realização dessa pesquisa, seguiu-se a Resolução do Conselho Nacional de Ética em Pesquisa (CONEP) №. 196, de 1996, e o. 251, de 1997, para pesquisas em seres humanos, do Ministério da Saúde ${ }^{(10)}$. O estudo foi aprovado pela Diretoria da Instituição e por seu Comitê de Ética em Pesquisa (Parecer 222/2006).

Os sujeitos foram convidados a participar da pesquisa, em seus turnos de trabalho; após esclarecimento dos objetivos da investigação e da livre participação, os que aquiesceram assinaram o consentimento livre e esclarecido. Em seguida procedeu-se a coleta de dados por meio da utilização de dois instrumentos: um questionário contendo 
informações relacionadas aos dados sóciodemográficos, além de outros sete itens relacionados a participação em treinamentos, percepção quanto às fases da SAE, participação e opinião quanto ao método; e uma escala intervalar do tipo Likert, com cinco pontos (variando de nunca a sempre), indicando a freqüência de sua participação nas diferentes atividades de enfermagem realizadas por estes profissionais e compatíveis com cada etapa da SAE. Para tanto, foram arrolados 110 itens considerados representativos das atividades cotidianas de enfermagem. Por exemplo, na fase de coleta de dados foi solicitado a cada sujeito que apontasse com que freqüência indaga o nome, procedência do paciente ou se ele faz uso de medicamentos; outros itens retratavam se ele avaliava temperatura, freqüência respiratória, presença de soroterapia, sondas dentre outros parâmetros. A lista de atividades foi elaborada pelas autoras. Os instrumentos foram avaliados quanto a sua aparência e conteúdo por um painel de três juizes com experiência em SAE (01 enfermeiro assistencial e 02 docentes de enfermagem). Foram aplicados a 07 sujeitos, não pertencentes à população, demonstrando serem adequados aos propósitos do estudo.

Para análise dos dados foi construído um banco de dados por meio do programa para microcomputador Statistical Package Social Science (SPSS), versão 10.0 Windows ${ }^{(11)}$.

\section{RESULTADOS E DISCUSSÃO}

Dos 77 sujeitos participantes do estudo, 90,9\% são auxiliares de enfermagem e 9,1\% técnicos de enfermagem, de unidades médica, cirúrgica e terapia intensiva, com faixa etária predominante entre 25 e 35 anos. Quanto ao gênero, $70,1 \%$ são do sexo feminino e $29,9 \%$ do sexo masculino. A maioria concluiu o curso há menos de 10 anos.

Embora as atribuições das categorias em estudo possam ser distintas para algumas das atividades analisadas, optou-se por considerar os dados em conjunto, tendo em vista o pequeno número de técnicos de enfermagem e pela similaridade de respostas em inúmeros itens; somente nas situações em que se observou representativa diferenciação entre as categorias funcionais, optou-se por apontálas na descrição dos resultados.

Em relação a utilização da SAE no setor em que trabalham, $72(93,5 \%)$ dos sujeitos reconhecem seu uso, sendo a maioria em um período de até cinco anos. Mas $75 \%$ dos prosissionais que referiram participar das fases da SAE na referida instituição, não conseguem identificar em qual ou quais fases participam.

O estudo revelou ainda que $74,0 \%$ dos sujeitos referiram ter recebido treinamento para participar da $\mathrm{SAE}$, especialmente após a formação profissional
$(67,5 \%)$. Apenas $46,8 \%$ dos sujeitos referiram saber quais são as fases da $\mathrm{SAE}$, dentre eles, $52,7 \%$ não souberam caracterizar as fases desse método.

Frente aos resultados, pode-se observar que os auxiliares e técnicos de enfermagem deste estudo não tiveram conteúdo sobre a SAE durante a sua formação e que passaram a conhecer essa metodologia de assistência após o ingresso profissional nas instituições, mediante treinamentos específicos. Contudo, trata-se de contingente de profissionais jovens, formados, em sua maioria há menos 10 anos, que reconhecem o uso desta metodologia e o seu benefício para a assistência.

$E$, muito embora os sujeitos tenham participado de treinamento específico sobre o tema no próprio hospital, ainda têm dificuldade em identificar as fases da SAE em que participam. O estudo alerta que os enfermeiros precisam estar preparados para incluir outros elementos da equipe de enfermagem na aplicação da SAE, na medida de suas possibilidades, para melhor entende-la e desenvolve-la em conjunto, tendo em vista que eles formam parte da equipe de enfermagem que assiste ao paciente ${ }^{(12)}$

Apesar de os sujeitos não relacionarem suas atividades às fases da SAE, descrevem, a seu modo, como atuam neste contexto, ou seja, realizando cuidados, promovendo conforto, seguindo normas, checando a prescrição de enfermagem, realizando admissão de pacientes, coletando informações, seguindo a prescrição de enfermagem e oferecendo apoio emocional e orientações.

Aproximadamente $70 \%$ dos sujeitos referiram não ter condições e $60 \%$ acreditam não ter aprovação do Conselho de Enfermagem para participarem das diferentes fases da SAE.

Apesar da falta de familiaridade com o método, é consenso para maioria dos entrevistados $(94,8 \%)$ que a SAE melhora a qualidade de sua assistência prestada ao paciente, muito embora não compreendam sua contribuição e sua participação na mesma.

A integração das diferentes categorias de enfermagem na realização da $\mathrm{SAE}$, respeitadas as exigências legais, a reflexão quanto à finalidade da SAE e a participação de cada um em suas fases consistem em algumas das estratégias para vencer barreiras da operacionalização deste método. Como já mencionado na literatura, há uma divisão do trabalho na enfermagem: de um lado o enfermeiro concebe o cuidado e do outro o auxiliar e o técnico o executam sem participarem do seu planejamento(12).

A legislação(5-6), por sua vez, incumbe privativamente ao enfermeiro a implantação, planejamento, organização e avaliação do processo de enfermagem; a lei do Exercício Profissional ${ }^{(7)}$ estabelece que: ao técnico de enfermagem compete executar atividades de nível médio, orientação e acompanhamento do serviço de enfermagem em 
grau auxiliar, e participar do planejamento da assistência de enfermagem. Os auxiliares de enfermagem exercem atividades de nível médio, de natureza repetitiva, sob supervisão, cabendo-lhes especialmente: observar, reconhecer e descrever sinais e sintomas ao nível de sua qualificação profissional entre outras atribuições.

Segundo os dados deste estudo, os auxiliares e técnicos de enfermagem participam direta ou indiretamente das diferentes fases da SAE, desempenhando atividades cotidianas, conforme se observa a seguir.

Em relação à Recepção de pacientes na unidade em que trabalham, $81,8 \%$ dos sujeitos referem receber pacientes para internação; 79,3\% recebem paciente durante retorno de exames; $87,0 \%$ recebem paciente que retornam do centro cirúrgico; $71,5 \%$ orientam pacientes e familiares quanto à rotina do hospital.

Como atividades relacionadas à Coleta de dados dos pacientes, $81,8 \%$ referiram que perguntam o nome; $65 \%$ indagam sobre antecedentes de patologias; $81,8 \%$ questionam sobre antecedentes alérgicos; $77,9 \%$ questionam o paciente sobre seu problema atual de saúde; $87,7 \%$ perguntam sobre o uso de medicamentos prescritos pelo médico e $52 \%$ questionam sobre automedicação. Aspectos relacionados à religiosidade do paciente nunca $(46,8 \%)$ ou raramente $(32,5 \%)$ são questionados por esses profissionais.

Quanto aos dados sobre respiração, 93,5\% observam a freqüência e ritmo; $93,5 \%$ observam a presença de dispnéia; 92,2\% observam a presença de tosse; $77,9 \%$ observam alterações na oxigeno terapia; $72,2 \%$ observam presença de coriza; $67,6 \%$ observam cateter nasal; $67,5 \%$ referem nunca realizar ausculta pulmonar.

Em relação à circulação, $89,6 \%$ verificam a pressão arterial; $81,8 \%$ observam presença de cateter venoso periférico ou central; $81,8 \%$ alterações na infusão venosa; 81,8 \% a freqüência de pulsos periféricos; $76,6 \%$ avaliam presença de edema; $76,6 \%$ avaliam perfusão periférica; 77,9\% verificam freqüência cardíaca, 67,5\% nunca realizam ausculta cardíaca e $57,1 \%$ não realizam a palpação de gânglios; 18,2\% sempre observam distensão de jugular.

Em relação à função neurológica $81,8 \%$ avaliam orientação no tempo e espaço; $87 \%$ referem avaliar alterações no nível de consciência. Quanto a avaliação de pupilas $23,4 \%$ realizam esta atividade; $33,8 \%$ avaliam acuidade visual, 55,9\% observam alterações nos olhos; $52 \%$ observam a acuidade auditiva.

A verificação da temperatura é realizada por $98,7 \%$ dos sujeitos e a higiene corporal por $89,6 \% \%$. Informam ainda que observam presença de lesões na pele $(90,9 \%)$; a freqüência e características da urina
$(85,7 \%)$; o tipo e aceitação da dieta $(83,1 \%)$; débito urinário $(80,5 \%)$; freqüência e características das fezes dos pacientes $(76,6 \%)$. Por outro lado, $85,7 \%$ não avaliam presença de ruídos abdominais e $81,8 \%$ referem não realizar percussão abdominal.

Em relação à locomoção dos pacientes, 63,7\% avaliam a necessidade de auxílio para deambulação (muletas, apoio); 57,2\% avaliam membros superiores quanto à simetria, contraturas, tremores; $58,5 \%$ avaliam membros inferiores quanto à simetria, contraturas e tremores; $28,6 \%$ avaliam o tipo de marcha do paciente, aspecto geralmente é pouco observado pela enfermagem.

Quanto ao aparelho reprodutor masculino e feminino, $80,5 \%$ dos sujeitos não avaliam a freqüência de atividade sexual e as alterações relacionadas à sexualidade; $67,5 \%$ nunca questionam sobre o uso de métodos contraceptivos; $76,6 \%$ nunca realizam exame das mamas; $62,3 \%$ nunca realizam exame dos genitais.

As alterações em drenos $(94,8 \%)$, em sondas $(97,4 \%)$ e em cateteres $(90,0 \%)$, presença e condições de curativos $(96,1 \%)$ são referidas como observadas por esses profissionais.

A presença de dor é questionada por 90,6\% dos sujeitos, assim como $74 \%$ questionam e observam sensação de medo e ansiedade relacionados aos procedimentos cirúrgicos, internação ou à doença; $72,7 \%$ observam expressão de medo e ansiedade dos pacientes.

A qualidade dos relacionamentos familiares nunca é indagada por 33,8\% dos sujeitos e 76,6\% não questionam sobre as práticas terapêuticas não medicamentosas.

Em relação à participação direta ou indireta dos técnicos e auxiliares na fase de Diagnóstico de Enfermagem, 85,7\% dos sujeitos informaram que nunca estabelecem o diagnóstico de enfermagem, mas $46,8 \%$ referem identificar o problema do paciente.

Quanto à observação e informação de sinais e sintomas que auxiliam na seleção dos diagnósticos de enfermagem, 57,2\% realizam esta atividade; $61,1 \%$ referem ler os diagnósticos de enfermagem listados no prontuário do paciente.

Em relação a atividades relacionadas à fase de Planejamento da Assistência de Enfermagem, $36,4 \%$ referem participar da seleção de cuidados de enfermagem para cada diagnóstico de enfermagem estabelecido; todavia, apenas $11,7 \%$ referiram fazer sugestões para a prescrição de enfermagem freqüentemente.

Em relação às atividades inerentes a fase de I mplementação da Assistência de Enfermagem, 97,4\% realizam o cuidado prescrito; $98,7 \%$ dos sujeitos checam a prescrição de enfermagem após realizar o cuidado prescrito; $20,8 \%$ referem que nunca realizam cuidados que não estão prescritos; 
$50,7 \%$ referem executar cuidados que não foram prescritos pela enfermeira e $77,9 \%$ referem anotar os cuidados realizados não prescritos pela enfermeira.

No que se refere à Avaliação, 45,5\% dos sujeitos mencionaram efetuar a evolução dos clientes; esses achados sugerem que eles consideram a evolução como sinônimo de avaliação. A observação de alterações durante a realização do cuidado e a comunicação destas para o profissional enfermeiro são realizadas por $94,8 \%$ e $76.6 \%$ descrevem o resultado do cuidado prestado. Contudo tais atividades não retratam a fase de avaliação do processo de enfermagem.

Em relação à Alta hospitalar, anotam condições de deambulação do paciente (75,3\%), presença de dreno, soro e sondas $(97,4 \%)$, presença de lesões e curativos $(96,1 \%)$, entrega de documentos e pertences $(64,9 \%)$ e acompanhamento de familiares ou representantes de instituições de destino $(63,6 \%)$; ainda, orientam sobre medicamentos, retorno e exames $(72,7 \%)$.

Os resultados do presente estudo evidenciam que a maioria não se considera em condições de participar das atividades das diferentes fases da SAE e não tem autonomia legal para desenvolvê-las. Por outro lado, apontam sua participação na coleta de dados, no desenvolvimento e checagem das atividades prescritas por enfermeiros, na elaboração do relatório das atividades e, ainda, colaboram no planejamento da assistência. Tais dados reforçam a opinião das autoras de que essas atividades necessitam análise à luz das funções que lhes são atribuídas pela legislação.

Nesse aspecto, cabe lembrar que a legislação citada aponta a possibilidade de participação do técnico e do auxiliar de enfermagem em algumas das etapas da SAE, mas não explicita quais as atividades que realmente cada categoria pode desenvolver. Cabe destaque à prescrição de enfermagem que é definida na legislação como de atribuição de enfermeiros; as demais merecem novas investigações quanto a quem compete seu desenvolvimento, com vistas a prepará-los adequadamente para desenvolver o que lhe for atribuído por lei.

A maioria dos sujeitos mencionou que a SAE melhora a qualidade da assistência, reiterando achados da literatura(13); todavia, alguns referiram desinteresse na implementação da prescrição de enfermagem, uma vez que compartilham pouco das informações sobre os pacientes; tal aspecto favorece a resistência dos auxiliares e técnicos de enfermagem em cumprir a prescrição de enfermagem, pois ela não reflete as reais necessidades dos clientes ${ }^{(14)}$. Contudo, no presente estudo, observou-se índices maiores de realização das atividades prescritas pelo enfermeiro que os apontados por outros autores ${ }^{(13)}$.
As respostas dos sujeitos evidenciam que eles desempenham importante contribuição nas fases desse método de assistência que retratam atividades previstas em lei como admissão, observação de algumas evidências clínicas, implementação de cuidados e sua documentação, isto é registro de diferentes aspectos da condição clínica dos pacientes como de intervenções realizadas. A propósito da importância dos registros feitos por esses profissionais, a literatura ${ }^{(15)}$ aponta que os mesmos favorecem informações da assistência prestada e asseguram comunicação entre os membros da equipe.

Quanto aos registros de enfermagem, na instituição onde se realizou o estudo, estão previstos dois tipos de registros em prontuários: a evolução de enfermagem, de responsabilidade exclusiva do enfermeiro e a anotação de enfermagem, de responsabilidade do auxiliar e ou técnico de enfermagem, dos procedimentos e reações apresentadas pelo paciente, de acordo com o que foi prescrito pelo enfermeiro. Quando os sujeitos afirmam que realizam a evolução do paciente, em função da falta de familiaridade com as fases da SAE e o propósito de cada uma delas, constatamos certa divergência entre as atividades de anotação ou relatório de enfermagem e a de evolução. Estas não retratam os diversos aspectos que compõem a fase de avaliação da SAE se considerarmos o proposto na literatura ${ }^{(16)}$ uma vez que, além da avaliação do resultado no estado do paciente decorrente da intervenção, essa fase congrega a análise do método empregado quanto à sua pertinência e conseqüência.

\section{CONCLUSÃO}

A participação dos auxiliares e técnicos de enfermagem nesta metodologia ainda é obscura; eles não percebem suas contribuições, fato reforçado pela compreensão dúbia da legislação; frente a isso, recomenda-se uma força tarefa para orientações sobre tais aspectos tanto ao enfermeiro, que é gerenciador dos cuidados de enfermagem, como à sua equipe. $O$ enfermeiro deve conhecer a interdependência das atividades realizadas na assistência ao paciente; ele é o responsável pela tomada de decisão quanto ao cuidado mas, não pode deixar de contar com a participação da equipe sob sua responsabilidade. Desse modo, de acordo com o nível de qualificação, os mesmos poderiam coletar dados, sendo que nesta pesquisa ficou evidenciado que eles já o fazem, subsidiar a assistência de enfermagem a ser implementada face aos diagnósticos de enfermagem estabelecidos pelo enfermeiro e ainda participar, junto com o enfermeiro, do planejamento da assistência enfermagem. Tais aspectos contribuíram, ainda, para que identificassem sua contribuição no cuidado ao paciente. 
O enfermeiro quando envolvido no proceso de formação de sua equipe deve rever sua atuação como educador uma vez que, para a implementação da SAE, faz-se necessária a participação destes profissionais.

Torna-se evidente a necessidade premente da inclusão deste tema na grade curricular dos cursos de auxiliares e técnicos de enfermagem, esclarecendo as atribuições e responsabilidades de cada membro da equipe de enfermagem.

\section{REFERÊNCI AS}

1. Jesus CAC. Assistência de enfermagem a clientes hematológicos: uma visão sistêmica. [dissertation]. Ribeirão Preto: Escola de Enfermagem/USP; 1992. $279 p$.

2. Garcia TR, Nóbrega MML, Carvalho EC. Nursing process: application to the professional practice. Online braz j nurs [Internet]. 2004;3(2). Avalaible from:

http://www.uff. br/nepae/siteantigo/objn302garciaetal. htm.

3. Mendes AA, Bastos MAR. Processo de enfermagem: seqüências no cuidar, fazem a diferença. Rev Bras Enferm. 2003; 56(3):271-6.

4. Carvalho EC, Bachion MM, Dalri MC, Jesus CAC. Obstáculos para implantação do processo de enfermagem no Brasil. Revista de enfermagem da UFPE On Line 2007; 1(1): 95-99.

5. Conselho Federal de Enfermagem. Resolução no 272 de 27 de agosto de 2002: Dispõe sobre a Sistematização da Assistência de Enfermagem - SAE - nas instituições de Saúde Brasileiras. Rio de Janeiro (Brasil): COFEN; 2002.

6. Conselho Regional de Enfermagem - CORENSP/DIR. Decisão no 008 de 1999. Dispõe sobre a Sistematização da Assistência de Enfermagem - SAE - nas instituições do Estado de São Paulo. São Paulo (Brasil). COREN-SP; 1999.

7. Conselho Regional de Enfermagem de São Paulo. Decreto 94.406 de 08 de junho de 1987. Regulamenta a Lei 7.498/86 e dispõe sobre a regulamentação do exercício da Enfermagem e dá outras providências. Documentos Básicos de Enfermagem: Enfermeiros, Técnicos e Auxiliares. São Paulo: Escrituras; 2001.

8.Gindri L, Medeiros HMF, Zamberlan C, Costenaro RGS. A percepção dos profissionais da equipe de enfermagem sobre 0 trabalho dos enfermeiros. Cogitare Enferm. 2005; 10(1): 34-41.

9.Tanaka LH, Leite MMJ. O cuidar no processo de trabalho do enfermeiro: visão dos professores. Rev Bras Enferm. 2007;60(6):681-6.

10. Ministério da Saúde; Conselho Nacional de Saúde. Resolução №196/96 - Normas regulamentadoras de pesquisa envolvendo seres humanos.. Brasília (Brasil): Ministério da Saúde; 1996.
11. SPSS Inc. SPSS for Windows [disk]. Version 10.0 Standard. Chicago (Illinois): SPSS Inc., 1999.

12. Peduzzi M, Anselmi ML. O processo de trabalho de enfermagem: cisão entre planejamento e execução do cuidado. Rev Bras Enferm. 2002; 55(4): 392-8.

13. Cogo ALP, Borba $E$ de. Prescrição de enfermagem: percepção do pessoal auxiliar de enfermagem de unidades médico-cirúrgicas em um hospital de ensino. Rev. gaúcha enferm. 1986; 7(1):61-77.

14. Ochoa-Vigo K, Pace AE, Santos CB. Análise retrospectiva dos registros de enfermagem em uma unidade especializada. Rev Latino-am Enferm. 2003; 11(2): 184-91.

15. Cianciarullo TI, Gualda DMR, Melleiro MM, Marina HA. Sistema de Assistência de Enfermagem: evolução e tendências. São Paulo: Ícone; 2001.

16. Alfaro-LeFevre R. Aplicação do processo de enfermagem: promoção do cuidado colaborativo. 5th ed. Porto Alegre: Artmed; 2005.

Artigo recebido em 20.02.08.

Aprovado para publicação em 31.03.09. 